\title{
Evolution of the Angle Between the Magnetic Moment and the Rotation Axis of Radio Pulsars
}

\author{
D. P. Barsukov, P. I. Polyakova, and A. I. Tsygan \\ Ioffe Physicotechnical Institute, Russian Academy of Sciences, \\ ul. Politekhnicheskaya 26, St. Petersburg, 194021 Russia \\ Received June 17, 2009; in final form, June 25, 2009
}

\begin{abstract}
It is shown that, when angular-momentum losses of a radio pulsar are represented as a sum of magnetic-dipole and current losses, the angle between the magnetic moment and rotation axis of the radio pulsar tends to some equilibrium value (near $45^{\circ}$ ). This process takes place on a timescale of the order of the pulsar's characteristic age. Taking into account the non-dipolarity of the pulsar's magnetic field changes this equilibrium angle.
\end{abstract}

PACS numbers: $97.60 . \mathrm{Gb}$

DOI: $10.1134 / \mathrm{S} 1063772909120075$

\section{INTRODUCTION}

The global structure of pulsar magnetospheres is being actively studied (see, e.g., [1-4]), but the braking mechanisms for radio pulsars and the evolution of the angle $\chi$ between the pulsar's magnetic moment and rotation axis remain open problems [5, 6]. Special interest in the latter problem has been generated by observations of the pulsars B1931+24 and B1822-09 [7, 8], in which we have probably observed changes in $\chi$.

The state of a pulsar is characterized by two vectors: the dipole moment of the magnetic field $\mathbf{m}$ and the angular velocity of the pulsar's rotation $\boldsymbol{\Omega}$. If $\mathbf{m}$ and $\boldsymbol{\Omega}$ are not parallel (i.e., the pulsar is not co-axial), there is a triad of linearly independent space vectors in the laboratory frame,

$$
\mathbf{m}, \quad \Omega, \quad[\Omega \times \mathbf{m}],
$$

which can be used as a basis [1] (Fig. 1). The torque applied to the pulsar can be written

$$
\mathbf{K}=K_{\Omega} \boldsymbol{\Omega}+K_{m} \mathbf{m}+K_{\perp}[\boldsymbol{\Omega} \times \mathbf{m}] ;
$$

the equation describing the rotation of a spherically symmetric neutron star,

$$
I \frac{d \mathbf{\Omega}}{d t}=\mathbf{K}
$$

then becomes

$$
\begin{gathered}
I \frac{d \Omega}{d t}=\left(\mathbf{K}, \frac{\Omega}{\Omega}\right)=K_{\Omega} \Omega+K_{m} m \cos \chi \\
I \Omega \frac{d \cos \chi}{d t}=K_{m} m \sin ^{2} \chi
\end{gathered}
$$

where $\chi$ is the angle between the vectors $\mathbf{m}$ and $\boldsymbol{\Omega}$ and $I$ is the moment of inertia of the neutron star. The direction of the evolution of angle $\chi$ is determined solely by the sign of $K_{m}$ [1].

\section{MAGNETIC-DIPOLE BRAKING MECHANISM}

We will first consider the magnetic-dipole braking mechanism for pulsars. In 1955, Deutsch [9] found the electromagnetic field around a conducting sphere

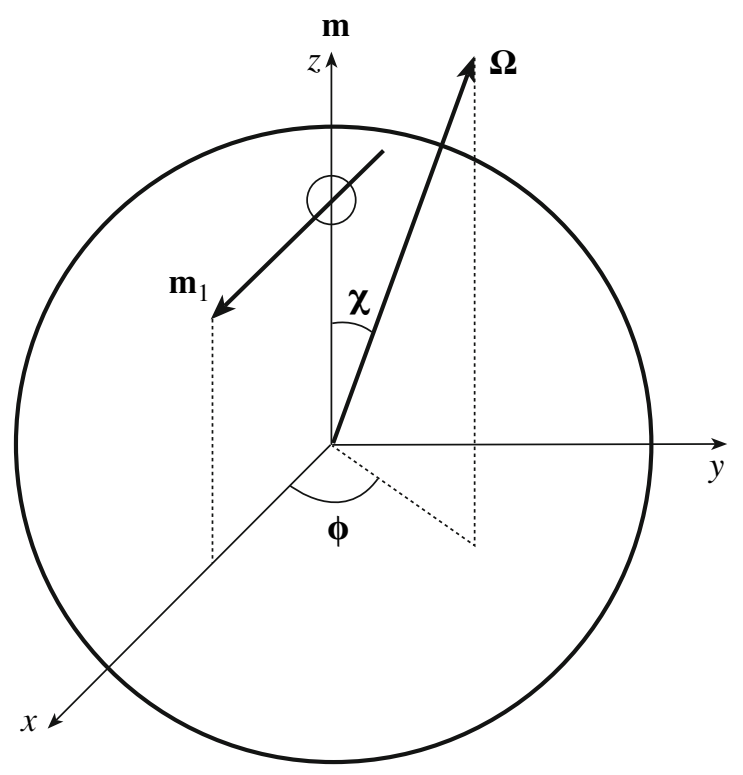

Fig. 1. Vectors and angles used in the pulsar coordinate system. 
with a magnetic dipole at its center that was rotating in a vacuum. Based on these results, Davis and Goldstein [10] considered the braking of a neutron star in vacuum in 1970. They found that, in the limit $\Omega a / c \ll 1$, the torque braking a neutron star is

$$
\begin{gathered}
\mathbf{K}_{d i p}=\frac{2}{3} \frac{\Omega^{2}}{c^{3}}[\mathbf{m} \times[\mathbf{m} \times \mathbf{\Omega}]] \\
+\frac{1}{a c^{2}}(\mathbf{m}, \boldsymbol{\Omega})[\mathbf{\Omega} \times \mathbf{m}]
\end{gathered}
$$

where $a$ is the neutron star radius. This expression differs from the angular-momentum losses for the rotation of a magnetic dipole in vacuum [11],

$$
\mathbf{K}_{\text {dip }}=-\frac{2}{3} \frac{\Omega^{2}}{c^{3}} m^{2} \boldsymbol{\Omega} \sin ^{2} \chi
$$

because of the appearance of boundary conditions at the surface of the conducting sphere.

It was assumed in [10] that the magnetic field inside the neutron star is uniform; i.e., it is created by a current at the surface of the neutron star. If surface currents are absent and the dipole magnetic field is created by volume currents inside the star, then the torque acting on the neutron star when $\Omega a / c \ll 1$ is [12]

$$
\begin{gathered}
\mathbf{K}_{d i p}=\frac{2}{3} \frac{\Omega^{2}}{c^{3}}[\mathbf{m} \times[\mathbf{m} \times \mathbf{\Omega}]] \\
+\frac{3}{5} \frac{1}{a c^{2}}(\mathbf{m}, \boldsymbol{\Omega})[\mathbf{\Omega} \times \mathbf{m}] .
\end{gathered}
$$

Melatos [12] considered not only the case $\Omega a / c \ll 1$, but also the case $\Omega a / c \sim 1$. Note that corrections to the braking torque related to non-dipolarity of the magnetic field are not small when $\Omega a / c \sim 1$.

According to (6) and (8), for a spherically symmetric neutron star, the evolution of angle $\chi$ is determined by $[1,10]$

$$
I \Omega \frac{d \chi}{d t}=-\frac{2}{3} \frac{\Omega^{3}}{c^{3}} m^{2} \cos \chi \sin \chi .
$$

It can also be readily shown using (6) and (8) that $[1,10]$

$$
\frac{\cos \chi}{P}=\text { const, }
$$

so that all pulsars are forced to become co-axial over a time of the order of the pulsar spin-down timescale [1, 5]. This appears to contradict observations (see, e.g., [13]).

Several ways of solving this problem have been proposed, in particular the following.

(a) All pulsars are born nearly orthogonal, and, since the angle changes very slowly in orthogonal pulsars, the observed pulsars simply have not had time to reach the regime $\chi=0[6,14]$. (b) All pulsars are born with the periods observed now; i.e., all observed pulsars are relatively young, and their rotation periods simply have not yet changed substantially during their lifetimes [6], so that the angle $\chi$ does not strongly differ from its initial value.

Goldreich [15] considered the case when a neutron star is strongly oblate. In this case, because of precession, the spin axis $\boldsymbol{\Omega}$ of the neutron star tends to align not with the magnetic moment $\mathbf{m}$ but with the neutron-star symmetry axis. If a neutron star is a triaxial ellipsoid, the angle $\chi$ permanently oscillates around some average value $\langle\chi\rangle \neq 0[12,16]$.

The very existence of the magnetic-dipole braking mechanism for pulsars was put in question in [17, 18 ], where it was shown that an orthogonal pulsar $(\chi=\pi / 2)$ is not spun down at all in a model with a force-free magnetosphere and the absence of current in the pulsar tube. This result was confirmed in [19] (see also [1]).

\section{CURRENT BRAKING MECHANISM}

There is a distinguished region in the magnetosphere of a pulsar, called the pulsar tube (a region of open lines of force), formed by magnetic-field lines that cross the light cylinder. It is generally accepted that, in the normal state of a radio pulsar, electric current flows in the pulsar tube. For the charge of the neutron star to be conserved, it is usually assumed that the same electric current flows back in a narrow layer near the pulsar tube boundary. These two currents close on the neutron star in the neighborhood of the polar cap. For these currents to be closed, some part of their path must cross the magnetic field. As a result, a Lorentz force induced by these currents, $\mathbf{F}=$ $\frac{1}{c}[\mathbf{j} \times \mathbf{B}]$, acts in the polar-cap region, and associated torques act on the neutron-star crust. An expression for this torque was obtained in [14]:

$$
\mathbf{K}_{c u r}=-\frac{2}{3} \frac{\Omega^{3}}{c^{3}} m^{2} \alpha \frac{\mathbf{m}}{m} \cos \chi .
$$

Here, the parameter $\alpha$ characterizes the magnitude of the electric current flowing through the pulsar tube:

$$
\begin{gathered}
\alpha=2 \cdot \frac{3}{4} \frac{j}{j_{G J}}\left(\frac{R_{t}(\eta)}{R_{0}(\eta)}\right)^{4}, \\
j_{G J}=\frac{\Omega B_{0}}{2 \pi} \cos \chi,
\end{gathered}
$$

where $j$ is the electric-current density in the pulsar tube, $j_{G J}$ the Goldreich-Julian current, $B_{0}$ the dipole magnetic field at the magnetic pole of the neutron star, $R_{t}(\eta)$ the pulsar-tube radius at height $\eta$, $R_{0}(\eta)=a \sqrt{(\Omega a / c)} \eta^{3 / 2}, \eta=r / a$, and $r$ is the distance from the center of the star. The factor of two allows for the contribution from both poles. 
Both the magnetic-dipole and current losses can be described by formula ( 1 ) of problem 2 in Section 72 of Landau and Lifshitz [11]. The total torque acting on a rotating neutron star, determined by the Maxwell stress tensor, is

$$
\begin{aligned}
\mathbf{K} & =\frac{r^{3}}{4 \pi} \int([\mathbf{n} \times \mathbf{E}](\mathbf{n E}) \\
& +[\mathbf{n} \times \mathbf{H}](\mathbf{n} \mathbf{H})) d \Omega,
\end{aligned}
$$

where $\mathbf{n}=\frac{\mathbf{r}}{r}, \mathbf{E}$ and $\mathbf{H}$ are the electric and magnetic field near the rotating neutron star, $r$ is the distance from the center of the star, $a$ is its radius, $a<r<\frac{c}{\Omega}$, and $d \Omega$ is an element of solid angle. The electriccurrent density in an axially symmetric pulsar tube is

$$
\mathbf{j}=-A(\xi) j_{G J} \frac{\mathbf{B}}{B} \text {. }
$$

The dimensionless variable $\xi(0 \leq \xi \leq 1)$ describes the position of a point inside the tube, $\xi=0$ corresponds to the tube center, and $\xi=1$ to its lateral surface. In the case of a purely dipolar magnetic field,

$$
\mathbf{B}=\frac{1}{r^{3}}(3 \mathbf{n}(\mathbf{n m})-\mathbf{m}),
$$

in a regime with a free outflow of charges from the surface, the factor $A$ in (14) is [20]

$$
A=1-\kappa,
$$

where $\kappa \approx 0.15$ describes the effect of frame dragging.

In a spherical coordinate system with the $O z$ axis aligned with the magnetic field, a thin tube with a current is described by the equations $\theta(\eta)=\theta_{0} \sqrt{\eta}$, $\phi=$ const, where $\theta_{0}=\sqrt{\Omega a / c} \ll 1$. According to the Stokes theorem, the Maxwell equations

$$
\operatorname{curl} \mathbf{H}=\frac{4 \pi}{c} \mathbf{j}, \quad \operatorname{div} \mathbf{H}=0
$$

lead to an expression for the magnetic-field component $H_{\phi}$

$$
H_{\phi}=\frac{2 \pi}{c} j a \theta(\eta) \eta \xi=-\frac{\Omega B_{0} a}{c} \xi \frac{\cos \chi}{\eta^{3}} \theta_{0} \eta^{\frac{3}{2}} A,
$$

where $a \theta(\eta) \eta=a \theta_{0} \eta^{3 / 2}$ is the tube radius a distance $r$ from the center of the star and $B_{0}=2 \mathrm{~m} / \mathrm{a}^{3}$ is the field intensity at the magnetic pole.

Substituting the total magnetic field $\mathbf{B}+H_{\phi} \mathbf{e}_{\phi}$ into (13) and taking into account that $\mathbf{B n} \approx B \cos \chi$ (for a thin tube), we obtain the expression for the current torque (the factor of two allows for the two poles):

$$
\mathbf{K}_{c u r}=2 \frac{r^{3}}{4 \pi} \int H_{\phi}\left[\mathbf{n} \times \mathbf{e}_{\phi}\right] d \Omega
$$

Taking into account that $d \Omega=\sin \theta d \theta d \phi \approx \theta d \theta d \phi$, $\left[\mathbf{n} \times \mathbf{e}_{\phi}\right]=-\mathbf{e}_{\theta}, \theta(\eta)=\theta_{0} \sqrt{\eta} \xi$, i.e., $d \Omega=\theta_{0}^{2} \eta \xi d \xi d \phi$, we substitute (18) into (19) and integrate over $\phi$ and $\xi$; this yields the expression for the vector $\mathbf{K}_{\text {cur }}$ :

$$
\mathbf{K}_{\text {cur }}=-2 \frac{\mathbf{m}}{m} \frac{B_{0}^{2} a^{3}}{8}\left(\frac{\Omega a}{c}\right) \theta_{0}^{4} A \cos \chi .
$$

The current torque $\mathbf{K}_{\text {cur }}$ does not depend on the height at which we have integrated over the tube cross section ( $r$ could be equal to $a$ ). However, when calculating $\mathbf{K}_{\text {cur }}$ for the distorted dipole magnetic field, we must take $r>a$ values where the field is nearly dipolar (where all the $l$-pole moments, except for $l=1$, are small). We prefer this way of obtaining the current torque $\mathbf{K}_{c u r}$, because it does not depend on the structure of the surface currents or the presence of a non-dipolar magnetic field at the surface of the neutron star.

According to (11), for a spherically symmetric neutron star, the evolution of angle $\chi$ is now determined by $[1,14]$

$$
I \Omega \frac{d \chi}{d t}=\frac{2}{3} \frac{\Omega^{3}}{c^{3}} m^{2} \alpha \cos \chi \sin \chi .
$$

We can readily show using (11) that [1]

$$
\frac{\sin \chi}{P}=\text { const, }
$$

so that all pulsars are forced to become orthogonal over a time of the order of the pulsar spin-down timescale $[1,5,6]$.

It is noteworthy that, in this case, the neutronstar asymmetry and associated precession of the spin axis do not prevent the pulsar achieving an orthogonal rotation regime [21].

\section{SHAPE AND RADIUS OF THE PULSAR TUBE CROSS-SECTION}

Jones [14] supposed that the contributions to the braking of a neutron star due to the magnetic-dipole and current mechanisms can be added. The total torque $\mathbf{K}$ applied to a neutron star is then

$$
\mathbf{K}=\mathbf{K}_{\text {dip }}+\mathbf{K}_{\text {cur }},
$$

and hence

$$
I \Omega \frac{d \chi}{d t}=-\frac{2}{3} \frac{\Omega^{3}}{c^{3}} m^{2}(1-\alpha) \cos \chi \sin \chi .
$$

In this case, stationary values of the angle $\chi$ different from $\chi=0^{\circ}$ and $\chi=90^{\circ}$ are possible only for $\alpha=1$. If $\alpha(\chi)$ decreases with increasing $\chi$, there is a stable stationary state at the point where $\alpha(\chi)=1$. Jones [14] assumed $\alpha \ll 1$, so that the pulsar reached a coaxial regime. 
An example when this cannot be done, and, moreover, there are no magnetic-dipole losses whatsoever, was demonstrated in [17, 19] (see also [1]). However, the results of observations of the pulsar B1931+24 [7] probably provide evidence not only that pulsars undergo magnetic-dipole losses, but also that these can be added to current losses. Here, we postulate that there exist both magnetic-dipole and current losses, whose contributions to the braking of a neutron star can be added.

Thus far, it has been supposed that the tube of open lines of force is axisymmetrical, i.e., its cross section at neutron-star surface (at its base) is a circle, whose radius does not depend on $\chi$. Of course, this is a good approximation for the axisymmetric case $\cos \chi= \pm 1$, when $\boldsymbol{\Omega}$ is parallel to $\mathbf{m}$.

Biggs [22] considered the dependence of the pulsar tube shape on $\chi$ in a vacuum magnetosphere model. It was shown that, as $\chi$ changed from $0^{\circ}$ to $90^{\circ}$, the pulsar tube cross section is flattened in the plane of the vectors $\boldsymbol{\Omega}$ and $\mathbf{m}$; i.e., in this direction, the size of the pulsar tube decreases from $\theta_{0} a \eta^{3 / 2}$ to $\theta_{1} a \eta^{3 / 2}$. At the same time, the size of the pulsar tube does not change in the direction of the vector $[\boldsymbol{\Omega} \times \mathbf{m}]$. If we suppose that the pulsar magnetic field remains purely dipolar up to the light cylinder, then

$$
\sin \theta_{1}=\sin \theta_{0} g(\chi)
$$

where we have introduced the notation

$$
\begin{gathered}
g(\chi)=\left(\frac{(1-\mu)^{3}}{1+3 \mu}\right)^{1 / 4} \\
\cos \chi=\frac{1-3 \mu}{\sqrt{1+3 \mu}} .
\end{gathered}
$$

As $\chi$ changes from 0 to $90^{\circ}, \mu$ varies from 0 to $\frac{1}{3}$; $\chi=90^{\circ}$ corresponds to $\mu=\frac{1}{3}$, and

$$
g\left(\frac{\pi}{2}\right)=\left(\frac{4}{27}\right)^{\frac{1}{4}} \approx 0.620 .
$$

This coincides with the results of [22] and differs from the value $g\left(\frac{\pi}{2}\right) \approx 0.54$ given in [13].

In the model [22], the pulsar tube cross section becomes similar to an ellipse; one of its axes does not change its length, while the other decreases by a factor of $g(\chi)$. The expression for $\alpha(\chi)$ can be written

$$
\alpha(\chi)=2 \frac{3}{4} A g(\chi)^{2} .
$$

Figure 2 shows the $\alpha(\chi)$ dependence for this case (solid curve). The thin horizontal line marks the value $\alpha=1$ required for the stationary state. There exists a stationary state at the angle $\chi \approx 48^{\circ}$, and, since

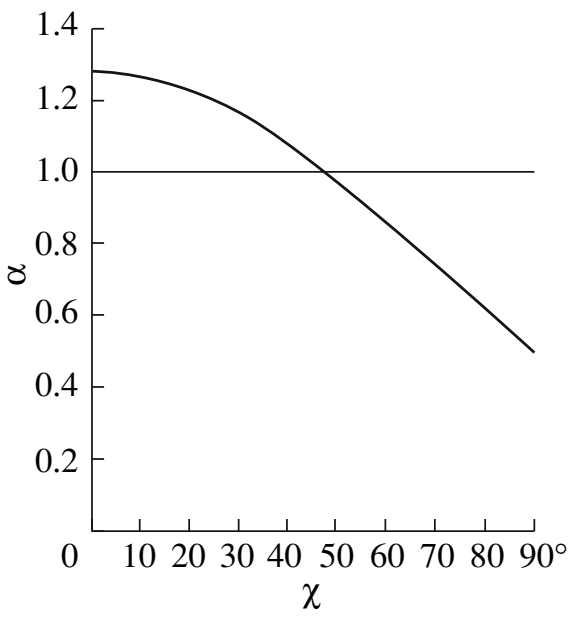

Fig. 2. Dependence of the function $\alpha(\chi)$ determining the current losses on the angle $\chi$ (solid curve). The thin horizontal line marks the value required for the stationary state, $\alpha=1$. There is a stable stationary state at the angle $\chi \approx 48^{\circ}$.

$\alpha(\chi)$ decreases with increasing $\chi$, this state is stable. The model predicts that pulsars should tend to the equilibrium angle $\chi \approx 48^{\circ}$, in contradiction with the observed angle distribution for pulsars (see, e.g., [13]).

If the size of the pulsar tube varies as is shown in [1, Fig. 2.17] or in [23] (see also [24]), according to our model for pulsars with purely dipolar magnetic fields, equilibrium angles $\chi$ do not exist.

\section{PRECESSION OF ANGULAR VELOCITY}

Let us consider precession of the angular velocity of rotation of a neutron star connected with the second term in (8). We assume that the neutron star is a perfectly rigid sphere. In this connection, we neglect the effect of asphericity of the neutron star (see, e.g., $[8,12,15]$ ). Also, for simplicity, we will not take into account dissipative processes related to the precession $[8,12,25]$.

Let us align the $O z$ axis with the magnetic moment $\mathbf{m}$. Equation (3) then becomes

$$
\begin{gathered}
\frac{I}{K_{0}} \frac{d \Omega}{d t}=-\left(\sin ^{2} \chi+\alpha \cos ^{2} \chi\right), \\
\frac{I}{K_{0}} \Omega \frac{d \cos \chi}{d t}=(1-\alpha) \sin ^{2} \chi \cos \chi, \\
\frac{I}{K_{0}} \Omega \frac{d \phi}{d t}=-\frac{9}{10} \frac{c}{\Omega a} \cos \chi,
\end{gathered}
$$

where $\phi$ is the azimuthal angle of the vector $\boldsymbol{\Omega}=$ $(\Omega \sin \chi \cos \phi, \Omega \sin \chi \sin \phi, \Omega \cos \chi)$, and $K_{0}=$ $2 \Omega^{3} m^{2} / 3 c^{3}$. Hence, the precession period is

$$
T_{p}=\frac{2 \pi}{\Omega_{p}}, \quad \Omega_{p}=-\frac{3}{5} \Omega \frac{m^{2}}{a c^{2} I} \cos \chi,
$$




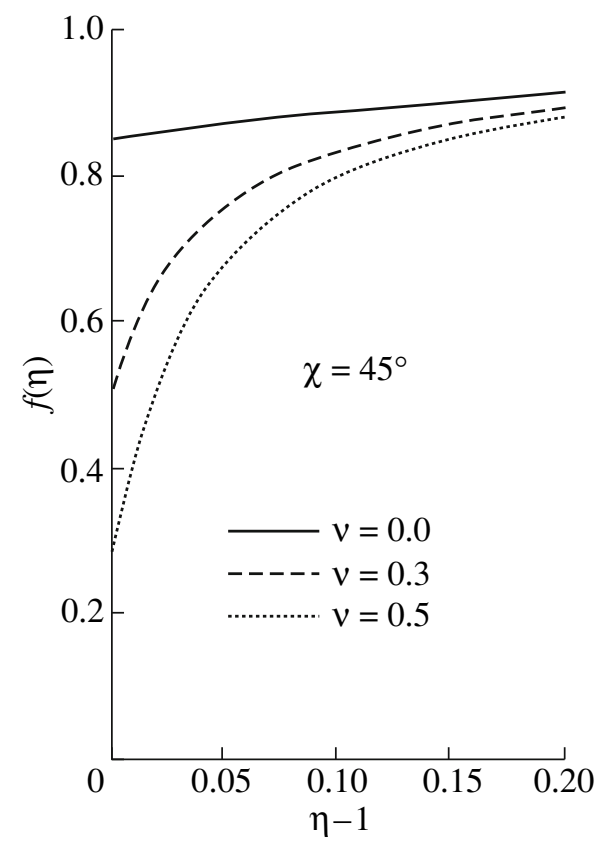

Fig. 3. The function $f(\eta)$ for $\gamma=\pi, \chi=45^{\circ}, \kappa=0.15$, $\Delta=0.1$. The solid curve shows the case of a purely dipolar magnetic field $(\nu=0)$, the dashed curve the case $\nu=0.3$, and the dotted curve the case $\nu=0.5$. The function $f(\eta)$ is everywhere positive and monotonically increasing.

and is much less than the pulsar age $\tau$ (i.e., the characteristic timescales for changes in $\Omega$ and $\chi$ ):

$$
\begin{gathered}
\frac{T_{p}}{\tau}=\frac{40 \pi}{9}\left(\frac{\Omega a}{c}\right) \frac{\sin ^{2} \chi+\alpha \cos ^{2} \chi}{\cos \chi} \ll 1, \\
\tau=\frac{P}{2 \dot{P}}=-\frac{\Omega}{2 \dot{\Omega}}
\end{gathered}
$$

(at least for sufficiently old pulsars with $P \gtrsim 1 \mathrm{~s}$ and for angles $\chi$ not too close to $90^{\circ}$ ). Asphericity of the neutron star's shape can result in a considerable decrease in the precession period.

Averaging (29) and (30) over the precession period, we obtain:

$$
\begin{gathered}
\frac{I}{K_{0}}\left\langle\frac{d \Omega}{d t}\right\rangle=-\left(\sin ^{2} \chi+\langle\alpha\rangle \cos ^{2} \chi\right), \\
\frac{I}{K_{0}} \Omega\left\langle\frac{d \cos \chi}{d t}\right\rangle=(1-\langle\alpha\rangle) \sin ^{2} \chi \cos \chi,
\end{gathered}
$$

where $\langle\alpha\rangle$ is the value of $\alpha$ averaged over the precession period (in contrast to $\Omega$ and $\chi$, the current in the pulsar tube and $\alpha$ can vary strongly during the precession period; see below).

The equilibrium value of $\chi$ is achieved if the mean value over the precession period $\langle\alpha(\chi)\rangle=1$. Thus, if the mean for the period $\langle\alpha(\chi)\rangle$ decreases with increasing $\chi$, this state is stable.
Note that, if the value of the angle $\chi$ corresponds to the equilibrium state $\langle\alpha(\chi)\rangle=1$, the rate at which the neutron star spins down does not depend on the equilibrium value of $\chi$ :

$$
\left.I \frac{d \Omega}{d t}\right|_{\langle\alpha\rangle=1}=-\frac{2}{3} \frac{\Omega^{3} m^{2}}{c^{3}} .
$$

The timescale for the stationary value of $\chi$ to be reached coincides to order of magnitude with the characteristic age of the pulsar,

$$
\tau=\frac{P}{2 \dot{P}}=\frac{3}{16 \pi^{2}} \frac{I c^{3} P^{2}}{m^{2}} \approx 5.2 \times 10^{7} P^{2} \mathrm{yrs}
$$

(for $B_{0}=10^{12} \mathrm{G}, a=10^{6} \mathrm{~cm}$, a neutron star mass $\left.M=2 \times 10^{33} \mathrm{~g}, I=0.4 M a^{2} \approx 0.8 \times 10^{45} \mathrm{~g} \mathrm{~cm}^{2}\right)$.

If a pulsar with the current period $P=1 \mathrm{~s}(\tau \approx$ $5.2 \times 10^{7}$ yrs $)$ was born rapidly rotating, e.g., with $P \approx 3 \times 10^{-2} \mathrm{~s}\left(\tau \approx 5.2 \times 10^{4} \mathrm{yrs}\right)$, it has already passed through several characteristic ages during its spin-down to $1 \mathrm{~s}$. To calculate the time evolution of $\chi$, we must jointly solve the system of equations (34), (35). This shows, in particular, that the evolution of the angle slows as it approaches the equilibrium value, so that the angle can continue to change when $P \sim 1 \mathrm{~s}$, even for pulsars that were born rapidly rotating.

\section{NON-DIPOLAR MAGNETIC FIELD}

As is noted above, the lines of force in the pulsar tube cross the surface of the light cylinder; therefore, an electric current can flow in the tube. Moreover, if there exists a region in the pulsar tube where the longitudinal electric field $E_{\|}=\mathbf{E B} / B$ is non-zero (the pulsar-diode region [26]), particles will be accelerated in this region. Below, we use the "inner gap" model, in which particle acceleration in the pulsar tube takes place near the surface of the neutron star.

It is currently accepted that the magnetic field at the neutron-star surface can strongly differ from a dipolar field. This is required, e.g., to explain the existence of long-period radio pulsars [27, 28]. It is also supposed that the magnetic field near the surface of the neutron star must differ very strongly from a dipolar field in order for radio pulsars to operate in the Ruderman-Sutherland model [29-31].

We will describe the non-dipolarity of the magnetic field using the model [32] (see also [33, 34]). We suppose that a neutron star with radius $a$ has a magnetic dipole moment $\mathbf{m}$ (the field at its magnetic pole is then $B_{0}=2 \mathrm{~m} / \mathrm{a}^{3}$ ). An additional magnetic dipole with magnetic moment $\mathbf{m}_{1}$ is placed a distance $a \Delta$ below the surface (deeper in the star) in the region of the neutron-star pole. The vector $\mathbf{m}_{1}$ is perpendicular to $\mathbf{m}$; the angle between $\mathbf{m}_{1}$ and the $(\boldsymbol{\Omega}, \mathbf{m})$ 


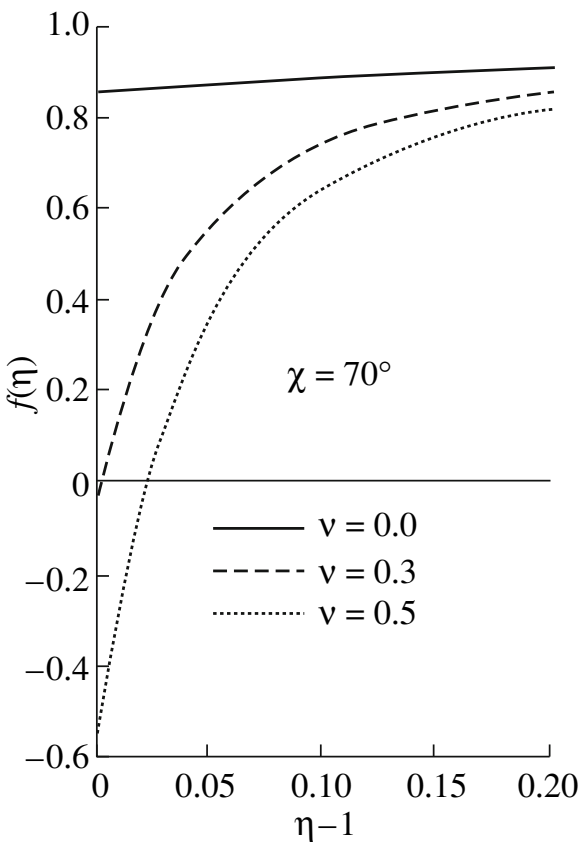

Fig. 4. Same as Fig. 3 for $\chi=70^{\circ}$.

plane is $\gamma$. We will assume everywhere that $\Delta$ is 0.1 , which approximately corresponds to the thickness of the neutron-star crust; this does not result in a rapid decay of the additional dipole $\mathbf{m}_{1}$. We introduce a spherical coordinate system $(\eta=r / a, \theta, \phi)$ with the $z$ axis directed along $\mathbf{m}$ and the $x$ axis along $\mathbf{m}_{1}$. Then, using the small-angle approximation $\theta \ll 1$, we can write for the total magnetic field $\mathbf{B}=\mathbf{B}_{0}+\mathbf{B}_{1}[32]$ :

$$
\begin{aligned}
B_{r}=\frac{B_{0}}{\eta^{3}}, \quad B_{\theta} & =\frac{B_{0}}{\eta^{3}}\left(\frac{\theta}{2}+\lambda \cos \phi\right), \\
B_{\phi} & =-\frac{B_{0}}{\eta^{3}} \lambda \sin \phi,
\end{aligned}
$$

where

$$
\begin{gathered}
\nu=\frac{B_{1}}{B_{0}}, \\
\lambda=\nu\left(\frac{\Delta \eta}{\eta-1+\Delta}\right)^{3}, \quad \eta=\frac{r}{a} .
\end{gathered}
$$

Note that, if the azimuthal angle $\phi$ is measured from the direction $O x$ in (31), then $\gamma=\pi-\phi$.

For simplicity, we assume that $\theta_{0} \ll \Delta$ and assume everywhere a thin-tube model. In this model for the magnetic field, we can assume to good accuracy that the Goldreich-Julian density $\rho_{G J}$ is constant over the tube cross section, and varies only with height $\eta$ (in particular, this means that we can neglect the Arons-Scharlemann term $\sim \theta_{0} \xi \sqrt{\eta} \sin \chi \cos \phi$ in $\left.\rho_{G J}\right)$ :

$$
\rho_{G J}=\frac{\Omega B(\eta)}{2 \pi c} f(\eta) \cos \chi
$$

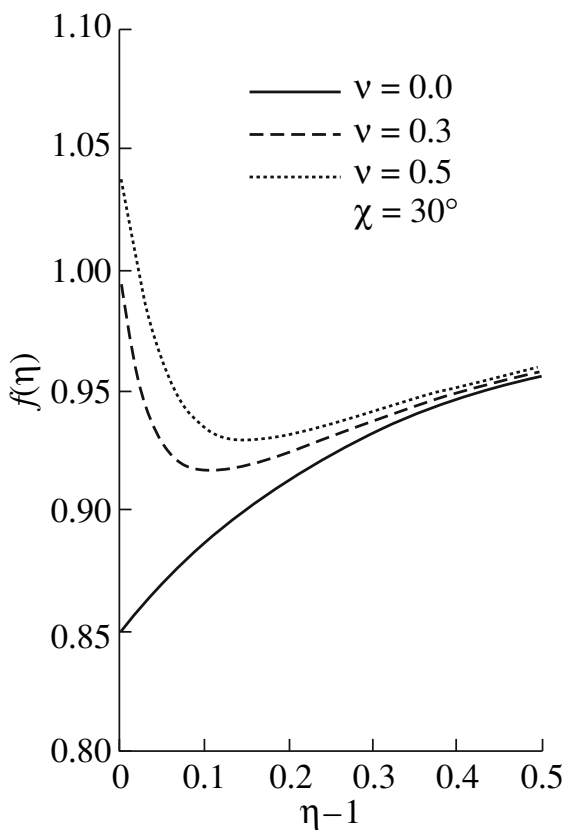

Fig. 5. Same as Fig. 3 for $\chi=30^{\circ}$. The function $f(\eta)$ becomes non-monotonic in the case of sufficiently strong non-dipolarity $(\nu=0.3$ and 0.5$)$.

where

$$
\begin{gathered}
f(\eta)=\frac{1}{\sqrt{1+\lambda^{2}}}\left[\left(1-\frac{\kappa}{\eta^{3}}\right)\right. \\
\left.+\lambda(\eta)\left(1+\frac{1}{2} \frac{\kappa}{\eta^{3}}\right) \tan \chi \cos \gamma\right] .
\end{gathered}
$$

If the lower plate of the diode is at a height $\eta_{0}$, the electric current flowing through the diode is [28]

$$
j=j_{G J} f\left(\eta_{0}\right) \text {. }
$$

In this case, the electrostatic potential $\Phi$ is [28]

$$
\begin{gathered}
\Phi=\frac{\Omega F}{2 \pi c}\left(f(\eta)-f\left(\eta_{0}\right)\right)\left(1-\xi^{2}\right) \cos \chi \\
\text { at } \eta_{0} \leq \eta \leq \eta_{c},
\end{gathered}
$$

where $F=\pi\left(\theta_{s} a\right)^{2} B$ is the magnetic flux through the tube and $\eta_{c}$ is the height of the upper plate of the diode (if the pulsar is off, $\eta_{c}=\infty$ ). For there to be no potential wells where particles can accumulate in the pulsar diode, the function $f(\eta)$ must increase as $\eta_{0} \leq \eta \leq \eta_{c}$.

To determine the height $\eta_{0}$ of the lower plate of the pulsar diode, we assume that

- the electric field at the diode plates is zero (then, in particular, (42) will be satisfied),

-the potential $\Phi$ monotonically increases in the pulsar diode,

- the pulsar diode is as close to the neutron star as possible. 

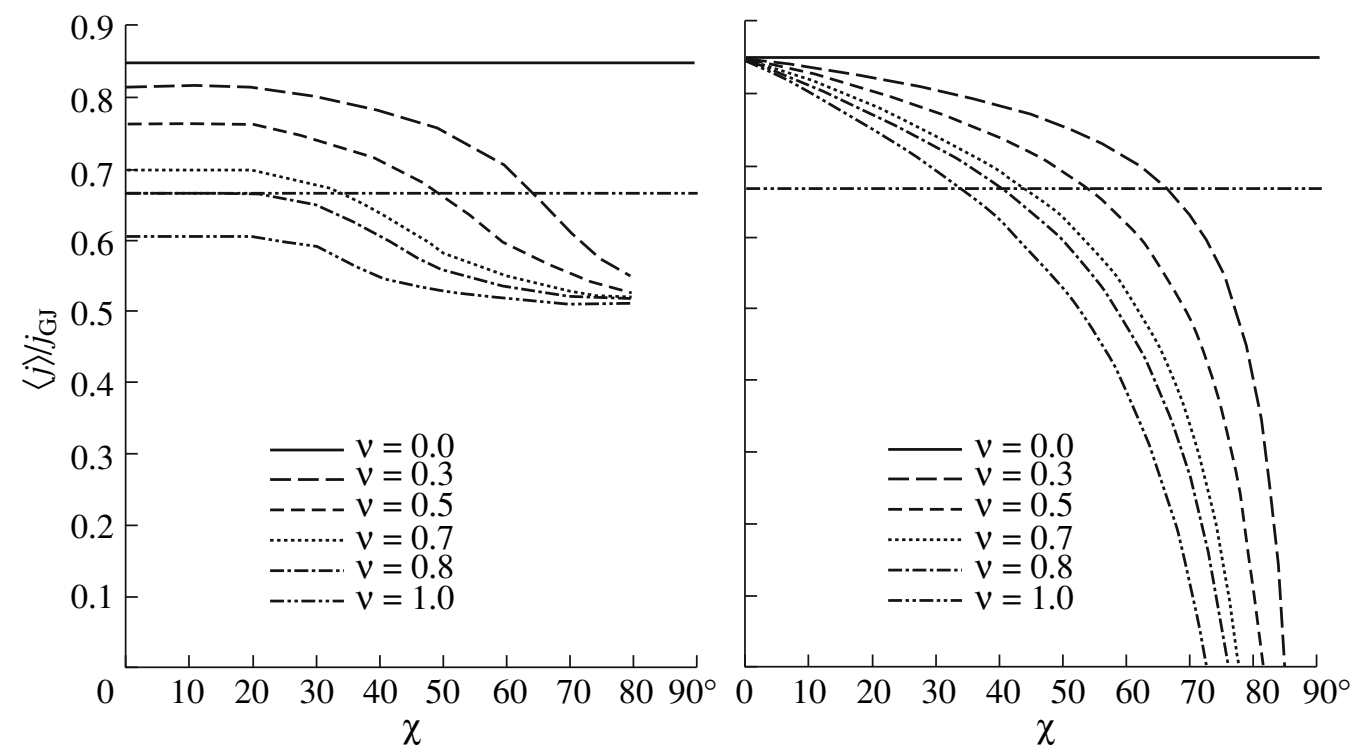

Fig. 6. Pulsar-tube current averaged over the precession period $\langle j\rangle$ as a function of the angle $\chi$; the right-hand plot corresponds to the approximate dependence according to (45). The solid curve shows a purely dipolar magnetic field $(\nu=0)$, the longdashed curve $\nu=0.3$, the short-dashed curve $\nu=0.5$, the dotted curve $\nu=0.7$, the dash-dot curve with one point $\nu=0.8$, and the dash-dot curve with two points $\nu=1.0$.
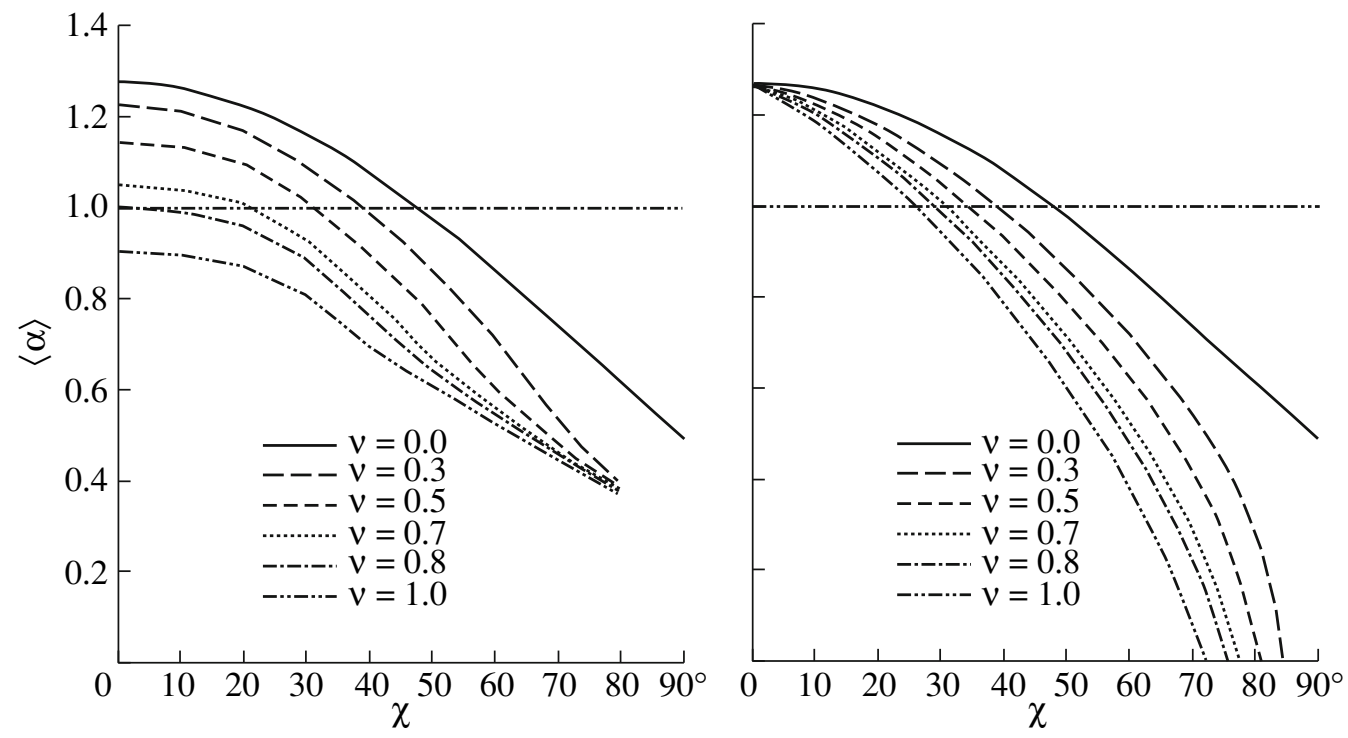

Fig. 7. The function $\langle\alpha(\chi)\rangle$ taking into account the dependence of the tube radius on $\chi$; the right-hand plot corresponds to the approximate dependence according to (45). The notation of the curves is the same as in Fig. 6.

Let us first consider the case $\cos \gamma<0$. Then, $f(\eta)$ is everywhere a monotonically increasing function (Fig. 3). Therefore, if $f(1) \geq 0$, the pulsar diode can be placed at the surface of the neutron star. Then, $\eta_{0}=1$, and the electron current density in the tube is $j=j_{G J} f(1)$.

However, a case is possible when $f(1)<0$ at the surface of the neutron star (Fig. 4). We can then assume that the entire region $f(\eta)<0$ is filled with stagnant protons or positrons (like those that are trapped in the region of closed lines of force). These are restrained from falling onto the neutron star by a very small electric field. For positive particles, this electric field compensates the force of gravity, while, for electrons, it (together with the gravitational field) forms a potential barrier, which must be overcome by electrons escaping from the neutron star if they are to reach the pulsar diode. Thus, the electron current is determined by the fraction of thermal electrons 

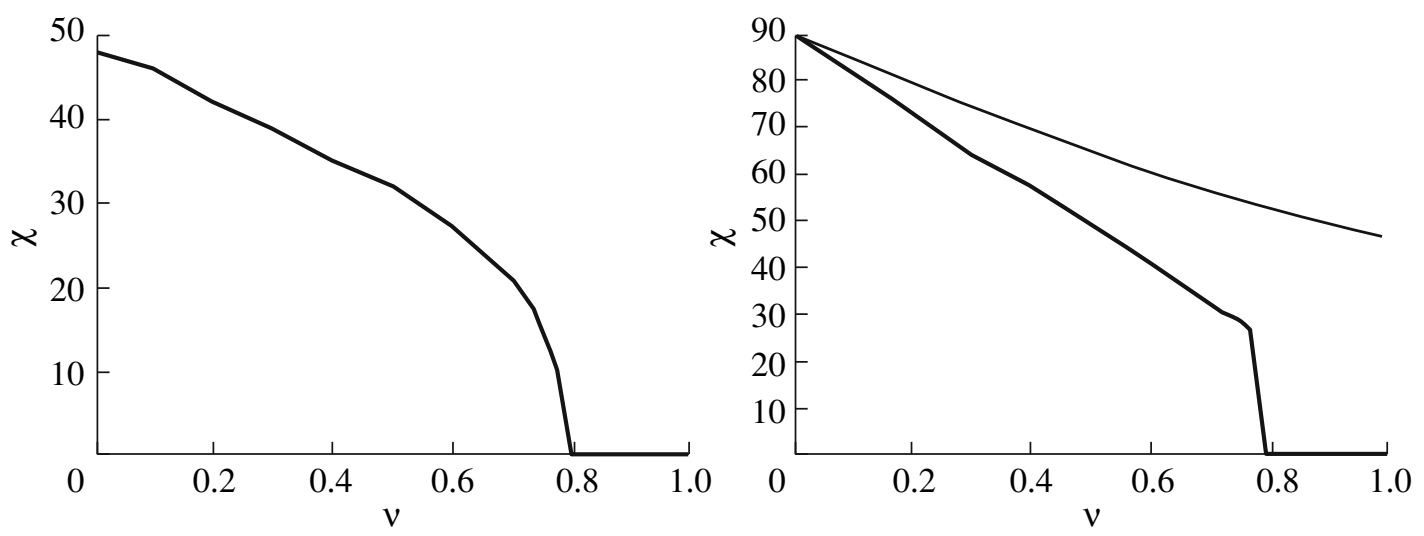

Fig. 8. The equilibrium angle $\chi$ as a function of the non-dipolarity $\nu$. The dependence of the tube radius on $\chi$ is taken into account in the left-hand graph. The dependence in the right-hand plot assumes that the pulsar-tube shape and radius do not depend on $\chi$. The current decreases at $\nu \geq 0.8$ such that the pulsar is forced to become co-axial. The dotted curve in the right-hand plot shows the equilibrium angle $\chi$ that would be obtained using (45).

that are capable of overcoming this potential barrier. Therefore, this current is very small, and and we can place the beginning of the pulsar diode at the point $f\left(\eta_{0}\right)=0$. In fact, the pulsar tube operates in the Ruderman-Sutherland regime and sparking is possible.

Let us now consider the case $\cos \gamma>0$. The function $f(\eta)$ is then positive everywhere, but it is no longer monotonic (Fig. 5). As a result, we cannot place the pulsar diode right at the neutron-star surface, since, in this case, or

- either $E_{\|}$is nonzero at the neutron-star surface,

— the potential $\Phi$ immediately becomes negative, preventing the escape of electrons.

Therefore, we place the pulsar diode at the minimum point of $f(\eta)$. In this case, electrons can escape from the neutron-star surface and $f\left(\eta_{0}\right) \approx 1$. At the diode base $\left(1<\eta<\eta_{0}\right)$, there is a region that is similar to the pulsar-tube bottom near the "outer gap." Here, either the potential oscillates (if the birth rate of electron-positron pairs is not high) [35] or regions with small enhancements of the potential (mini-diodes) alternate with regions where the longitudinal electric field is zero, and a medium of stagnant electrons is present (if electron-positron pairs are born very intensely). However, we cannot rule out the possibility that nearly the entire tube is filled with a medium of stagnant electrons, and there is an "outer gap" at heights $\eta \sim \eta_{L C}=c / \Omega a$, where the electric field is directed away from the star. Obviously, protons cannot traverse the huge potential barrier at the base of the "outer gap" $\left(1<\eta<\eta_{0}\right)$, and consequently $j=0$. However, electrons falling downward can be accelerated in this diode [36], i.e., $j<0$.
In the model considered, the electric current flowing through the pulsar tube can be approximately described by

$$
\begin{gathered}
j=j_{G J} \cdot \max \{0,1+\nu \tan \chi \cos \gamma\} \\
\text { at } \quad \cos \gamma<0, \\
j=j_{G J} \quad \text { at } \quad \cos \gamma>0 .
\end{gathered}
$$

During the precession time $T_{p}$, the angle $\chi$ remains virtually constant, and the angle $\gamma$ changes from 0 to $2 \pi$. As a result, the current in the pulsar tube at $\nu \tan \chi<1$ averaged over the precession period is

$$
\langle j\rangle=j_{G J}\left(1-\frac{\nu}{\pi} \tan \chi\right) .
$$

When averaging, we assumed that the non-dipolarity $\nu$ is the same for both pulsar tubes. At the same time, the angle $\gamma$ can be arbitrary. If $\nu \tan \chi>1$, the pulsar spends some time in a quasi-Ruderman regime during the precession interval.

Figure 6 presents the current in the pulsar tube averaged over the precession time $\langle j\rangle$ as a function of $\chi$. Figure 7 shows the a plot of the function $\langle\alpha(\chi)\rangle$, with the dependence of the tube radius on $\chi$ taken into account. When $\nu \geq 0.8$, the current is always less than $\frac{2}{3} j_{G J}$ (Fig. 7), and, accordingly, $\langle\alpha\rangle \leq 1$; i.e., the pulsar inevitably becomes co-axial.

The left-hand plot of Fig. 8 presents the equilibrium angle $\chi$ as a function of the non-dipolarity $\nu$. This dependence takes into account the change in the tube shape with the change in $\chi$, according to (28). This figure shows that there should be pulsars with equilibrium angles $\chi$ greater than $48^{\circ}$. Moreover, the most range of angles that is most populated in pulsars, $\chi \sim 10^{\circ}-30^{\circ}$ [13], corresponds to a very narrow interval of the magnetic-field non-dipolarity, 
$\nu \sim 0.7-0.8$. The absence of pulsars with equilibrium angles $\chi$ greater than $48^{\circ}$ is due to the sharp decrease in the pulsar-tube size in the model [22]. If we suppose that the pulsar-tube area does not change as a pulsar approaches the orthogonal regime or, as in the case presented in Fig. 2.17 of [1], this area increases, pulsars with purely dipolar magnetic fields $\nu=0$ will have equilibrium angles $\chi=90^{\circ}$.

The right-hand plot of Fig. 8 shows the equilibrium angle $\chi$ as a function of the non-dipolarity $\nu$ assuming that the shape and radius of the pulsar tube do not depend on $\chi$. The range of equilibrium angles $\chi \sim 10^{\circ}-30^{\circ}$ corresponds to a still narrower interval of the non-dipolarity $\nu$. Note that, with such strong non-dipolarity $(\nu \geq 0.7)$, the current losses become less than the magnetic-dipole losses; consequently, the mechanism for the stabilization of the angle $\chi$ related to the nonaxially symmetric shape of the neutron star probably operates [12, 15]. Moreover, it seems quite reasonable to suppose that, since the magnetic field itself induces a deformation of the neutron star [15], the axis for this deformation does not differ too strongly from the direction of $\mathbf{m}$, and precisely this mechanism provides equilibrium angles $\chi \sim 10^{\circ}-30^{\circ}$ for the majority of pulsars.

\section{ACKNOWLEDGMENTS}

The authors thank A.I. Chugunov, M.E. Gusakov, M.V. Ulanov, Yu.A. Shibanov, A.A. Danilenko, and D.A. Zyuzin for their support and valuable comments. This work was supported by the Program of State Support for Leading Scientific Schools of the Russian Federation (grant no. NSh-2600.2008.2). The authors are grateful to the referee for a careful analysis of the article and helpful criticism.

\section{REFERENCES}

1. V. S. Beskin, Axisymmetric Stationary Flows in Astrophysics (Fizmatlit, Moscow, 2006) [in Russian].

2. A. N. Timokhin, Mon. Not. R. Astron. Soc. 368, 1055 (2006).

3. A. N. Timokhin, arXiv: astro-ph/0607165v2 (2006).

4. I. Contopoulos and A. Spitkovsky, Astrophys. J. 643, $1139(2006)$.

5. S. A. Eliseeva, S. B. Popov and V. S. Beskin, arXiv: astro-ph/0611320 (2006).

6. V. S. Beskin and E. E. Nokhrina, Astrophys. Space Sci. 308, 569 (2006).

7. A. V. Gurevich and Ya. N. Istomin, Mon. Not. R. Astron. Soc. 377, 1663 (2007).

8. Ya. N. Istomin and T. V. Shabanova, Astron. Zh. 84, 139 (2007) [Astron. Rep. 51, 119 (2007)].
9. A. J. Deutsch, Ann. d'Astrophys. 18, 1 (1955).

10. L. Davis and M. Goldsteinm, Astrophys. J. 159, L81 (1970).

11. L. D. Landau and E. M. Lifshitz, Course of Theoretical Physics, Vol. 2: The Classical Theory of Fields (Nauka, Moscow, 1988; Pergamon, Oxford, 1975).

12. A. Melatos, Mon. Not. R. Astron. Soc. 313, 217 (2000).

13. I. F. Malov, Radiopulsars (Nauka, Moscow, 2004) [in Russian].

14. R. V. Jones, Astrophys. J. 209, 602 (1976).

15. P. Goldreich, Astrophys. J. 160, L1 1 (1970).

16. A. Melatos, Mon. Not. R. Astron. Soc. 288, 1049 (1997).

17. V. S. Beskin, A. V. Gurevich, and Ya. N. Istomin, Zh. Eksp. Teor. Fiz. 85, 401 (1983) [Sov. Phys. JETP 58, $235(1983)]$.

18. V. S. Beskin, A. V. Gurevich, and Ya. N. Istomin, Astrophys. Space Sci. 102, 301 (1984).

19. L. Mestel, P. Panagi, and S. Shibata, Mon. Not. R. Astron. Soc. 309, 388 (1999).

20. A. G. Muslimov and A. I. Tsygan, Mon. Not. R. Astron. Soc. 255, 61 (1992).

21. V. S. Beskin, A. V. Gurevich, and Ya. N. Istomin, Physics of Pulsar Magnetosphere (Cambridge Univ., Cambridge, 1993).

22. J. D. Biggs, Mon. Not. R. Astron. Soc. 245, 514 (1990).

23. J. Dyks, A. K. Harding, and B. Rudak, Astrophys. J. 606, 1125 (2004).

24. R. Narayan and M. Vivekanand, Astron. Astrophys. 113, L3 (1982).

25. P. B. Jones, Astrophys. Space Sci. 45, 369 (1976).

26. J. Arons and E. T. Scharlemann, Astrophys. J. 231, 854 (1979).

27. J. A. Hibschman and J. Arons, Astrophys. J. 554, 624 (2001).

28. E. M. Kantor and A. I. Tsygan, Astron. Zh. 80, 665 (2003) [Astron. Rep. 47, 613 (2003)].

29. M. A. Ruderman and P. G. Sutherland, Astrophys. J. 196, 51 (1975).

30. J. Gil and D. Mitra, Astrophys. J. 550, 383 (2001).

31. J. A. Gil, G. I. Melikidze, and D. Mitra, Astron. Astrophys. 388, 256 (2002).

32. V. D. Pal'shin and A. I. Tsygan, Preprint Fiz.-Tekhn. Inst. No. 1718 (FTI, St. Petersburg, 1998).

33. D. P. Barsukov, E. M. Kantor, and A. I. Tsygan, Astron. Zh. 83, 184 (2006) [Astron. Rep. 50, 159 (2006)].

34. D. P. Barsukov, E. M. Kantor, and A. I. Tsygan, Astron. Zh. 84, 1 (2007) [Astron. Rep. 51, 469 (2007)].

35. S. Shibata, Mon. Not. R. Astron. Soc. 287, 262 (1997).

36. K. Hirotani and S. Shibata, Astrophys. J. 564, 369 (2002).

Translated by G. Rudnitskii 